\title{
Transmission Power Management for IR-UWB WSN Based on Node Population Density
}

\author{
Fernando Ramírez-Mireles \\ Instituto Tecnológico Autónomo de México (ITAM) \\ Río Hondo 1, Col. Tizapán San Angel, \\ México City, D.F. C.P. 01000, \\ México \\ ramirezm@iee.org \\ http: // www.geocities.com / f _ ramirez _ mireles
}

\begin{abstract}
We propose a method to manage transmission power in nodes belonging to a wireless sensor network (WSN). The scenario contemplates uncoordinated communications using impulse radio ultra wideband (IR-UWB). Transmission power is controlled according to the statistical nature of the multiple access interference (MAI) produced by the nodes in the close vicinity of the communicating nodes. The statistical nature of the MAI is a function of the node population density within the area of coverage of the WSN. We show that when the node population density is high enough transmission power savings are possible.
\end{abstract}

Keywords: UWB, impulse radio, ad hoc networks, sensor networks.

\section{Introduction}

Wireless ad hoc networks (WAHN) are flexible networks for which there is no need of a central coordinator and for which the numbers of nodes and the topology of the network are not predetermined. A WSN is a type of WAHN composed of nodes with sensing capability. There are important differences between WSN and WAHN [1]. WSAN have a larger number of nodes and are deployed in close proximity to the phenomena under study, the nodes mainly use a broadcast communication paradigm and the topology of the network can change constantly due, for example, to nodes prone to fail (usully nodes have limited power, computational capabilities and memory).

The UWB is an indoor communication technique currently under intense research activity [2] due to many attributes, ${ }^{1}$ including its robustness against multipath conditions, its high capacity in a multiple access environment, the capability to achieve high transmission rates using a low amount of power, and, for pulse based UWB, the possibility of operating using a carrier-less modulation.

${ }^{1}$ According to [3] a signal is considered of UWB nature if the $10 \mathrm{~dB}$ bandwidth of the signal is at least $20 \%$ of its center frequency, or if this $10 \mathrm{~dB}$ bandwidth is at least $500 \mathrm{MHz}$. 
In particular, IR UWB [4] uses communications signals composed of ultra short pulses, and multiple access is achieved providing nodes with different spreading codes. The IR-UWB has been proposed for WAHN [5]. The many desirable characteristics of IR-UWB can be used in WAHN and WSN for simultaneous communication, ranging and positioning [6].

In this work we propose a method to manage the transmission power of a transmitting node. Communication signals use binary pulse position modulated (PPM) for data modulation and time hopping (TH) for code modulation. Spread spectrum multiple access (SSMA) is achieved using different TH codes for different users. We consider a WSN where the nodes know the spreading codes of their close neighbors, and the nodes communicate with the other nodes broadcasting messages. There can be some coordination between the nodes, e.g., they know the spreading codes of their close neighbors, and they can estimate roughly how many nodes are within their own neighborhood, but transmission are uncoordinated, e.g. with asynchronous transmission times and no power control.

Multiple nodes broadcasting their messages will generate MAI to other nodes, and this MAI will degrade the performance of the communication links. To preserve battery life, transmission power is controlled according to the statistical nature of the MAI produced by the nodes in the close vicinity of the communicating nodes. The statistical nature of the MAI is a function of the node population density within the area of operation of the WSN. We show that when the node population density is high enough transmission power savings are possible.

\section{MAI statistical nature and its impact on performance}

Consider a system with $k=1,2,3, \ldots, N_{u}$ nodes, and that nodes $2,3, \ldots, N_{u}$ are simultaneously broadcasting to other nodes. In particular, suppose that node 2 is attempting to transmit to node 1 , while the others $N_{u}-2$ nodes are broadcasting in the vicinity of node 1 . Lets denote the received signal power at node 1 as $\left(Q_{k, 1}\right)^{2} P_{k}, k=2,4, \ldots, N_{u}$, where $P_{k}$ is the transmitted power of node $k$ and $\left(Q_{k, 1}\right)^{2}$ is a factor (that can be random) reflecting the attenuation from transmitter $k$ to receiver 1 . If we ignore the effects of the noise, the signal-tointerference-ratio (SIR) at the input of node one's correlation receiver is

$$
\operatorname{SIR}_{\mathrm{in}}\left(N_{u}\right)=\frac{\left(Q_{2,1}\right)^{2} P_{2}}{\sum_{k=3}^{k=N_{u}}\left(Q_{k, 1}\right)^{2} P_{k}} .
$$

The statistical nature of the MAI at the output of the correlation receiver depends on the numbers of nodes $N_{u}-2$ producing it and also on the signal structure. In our case we consider binary TH-PPM signals ${ }^{2}$

$$
S^{(k)}(t)=\sum_{m=0}^{N_{s}-1} p\left(t-m T_{f}-c_{m}^{(k)} T_{c}-T_{d} d_{\left\lfloor m / N_{s}\right\rfloor}^{(k)}\right),
$$

$\overline{2}$ This signal set and its performance under different scenarios are studied in detail in [7] [8]. 
where $T_{f}$ is the frame time, $T_{c}$ is the code time shift, and $T_{d}$ is the data time shift. The $c_{m}^{(k)}$ is the pseudorandom time-hopping sequence for user $k$ with a range $0 \leq c_{m}^{(k)}<N_{h}$ and with sequence period $N_{p}$. The $d_{\left\lfloor m / N_{s}\right\rfloor}^{(k)}$ is the data of user $k$ that can be 0 or 1 and changes every $N_{s}$ hops (see fig. 1 ). Note that the data symbol changes every $N_{s}$ frames, therefore the symbol duration is $N_{s} T_{f}$ and the bit rate is $R=\frac{1}{N_{s} T_{f}}$.

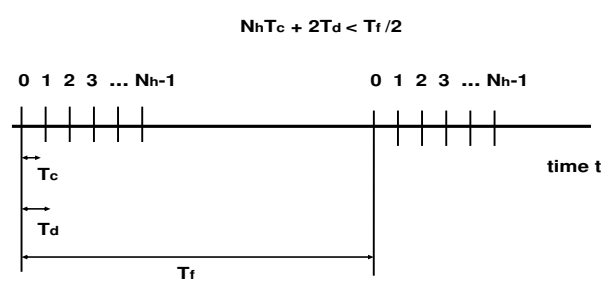

Fig. 1. Relation of TH-PPM parameters.

We consider two forms of pulse shape, with pulse parameters shown in table 1. The first is the second derivative of a Gaussian pulse

$$
p(t)=\left[1-4 \pi\left[\frac{t}{t_{n}}\right]^{2}\right] \exp \left(-2 \pi\left[\frac{t}{t_{n}}\right]^{2}\right)
$$

for $\quad-T_{w} / 2 \leq t \leq T_{w} / 2$, where $t_{n}=0.2877 \mathrm{~ns}$ is a parameter that determine the pulse duration $T_{w} \simeq 0.7$ ns. The second pulse shape is based on a gated sine wave

$$
p(t)=\sin \left(2 \pi \frac{Q}{T_{w}} t\right)
$$

for $-T_{w} / 2 \leq t \leq T_{w} / 2, T_{w}=2.0 \mathrm{~ns}$, where $Q=10$ is a positive integer, resulting in a signal spectrum centered at $\frac{Q}{T_{w}}=5 \mathrm{GHz}$. [7] [8]

For the signals in (2) the SIR at the output of the correlator can be written

$$
\operatorname{SIR}_{\text {out }}\left(N_{u}\right)=\frac{\left(Q_{2,1}\right)^{2} P_{2} A_{2,1}}{\sum_{k=3}^{k=N_{u}}\left(Q_{k, 1}\right)^{2} P_{k} C_{k, 1}} .
$$

where $A_{2,1}$ and $C_{k, 1}$ are normalized autocorrelation and cross correlation factors. The $A_{2,1}$ is produced by the desired signal and the $C_{k, 1}$ by the interference.

Given a pulse shape, the parameters mainly determining the MAI statistical nature are the number of pulses per symbol $N_{s}$, and the frame time between pulse transmissions $T_{f}$. It has been verified that under certain conditions (e.g. if $N_{s}$ is large enough), an increase of $N_{u}$ will cause the statistical nature of the 
Table 1. Values for TH-PPM time parameters.

\begin{tabular}{||c|c|c||}
\hline Parameters & Gaussian Pulse & Gated sine wave \\
\hline$T_{w}$ & $0.7 \mathrm{~ns}$ & $2.0 \mathrm{~ns}$ \\
$T_{f}$ & $70,150,250 \mathrm{~ns}$ & $200 \mathrm{~ns}$ \\
$T_{d}$ & $0.156 \mathrm{~ns}$ & $0.0995 \mathrm{~ns}$ \\
$T_{c}$ & $0.9 \mathrm{~ns}$ & $0.1 \mathrm{~ns}$ \\
$N_{h}$ & 8 & 200 \\
\hline
\end{tabular}

MAI to tend to Gaussianity [9]. However, when those conditions are not met, the MAI will have a probability density function that in general has thicker tails than the Gaussian distribution [10], producing a higher bit error rate (BER) than the Gaussian MAI, for the same SIR value.

Fig. 2 shows the difference in performance between a MAI that is not Gaussian (proposal approximation (PA)) and a MAI that is Gaussian (Gaussian approximation (GA)). Notice that as $N_{u}$ is increased the BER is degraded since the SIR in (5) decreases. Also notice that as $N_{u}$ is increased the PA tends to behave like the GA [9] [11] due to the central limit theorem in probability [12]. Fig. 2 verifies that BER for PA is higher than for GA, and shows the SIR gap between PA and GA.

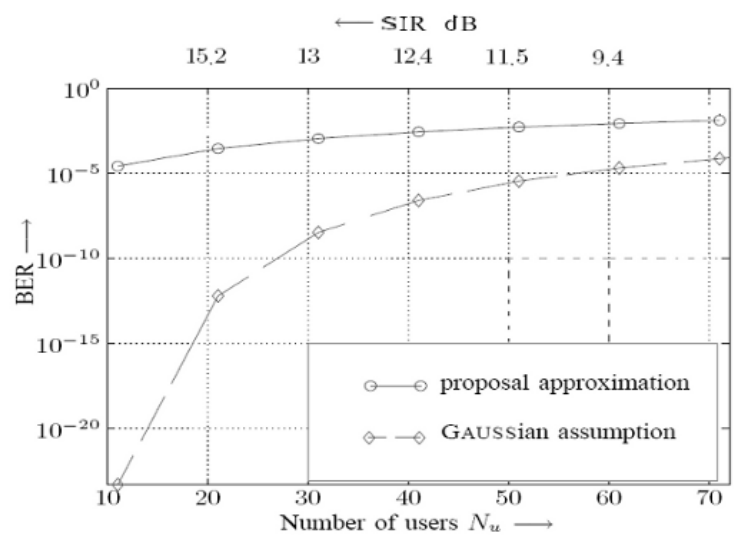

Fig. 2. BER for $T_{f}=100 \mathrm{~ns}, N_{s}=4$, and $10 \leq N_{u} \leq 70$ (elaborated in part with data taken from $[11])$. 


\section{Using the MAI statistical nature to control transmission power}

Each component of the MAI at the output of the correlation receiver is a random variable (r.v.) that depends on other r.v.'s such as the transmissions delays, the spreading codes, the users data, and the channel statistics. The MAI can be written as [7] [8]

$$
\begin{aligned}
\alpha & =\sum_{k=3}^{N_{u}} \alpha^{(k)} \\
\alpha^{(k)} & \triangleq \sum_{k=3}^{N_{u}} \sum_{m=0}^{N_{s}-1} A^{(k)}\left[R\left(\lambda_{m}^{(k)}\right)-R\left(\lambda_{m}^{(k)}-T_{d}\right)\right],
\end{aligned}
$$

where $\lambda_{m}^{(k)}=\left[c_{m-\Phi}^{(k)}-c_{m}^{(1)}\right] T_{c}+d^{(k)} T_{d}+\tau_{k}$ is a weighted sum of uniformly distributed r.v.s $c_{j}^{(k)}, d_{\left\lfloor m / N_{s}\right\rfloor}^{(k)}$ and $\tau_{k}, R(\cdot)$ are cross correlation terms, $\tau_{k}$ are random transmission delays, and $\Phi$ is a r.v. that depends on the transmission delays. We notice that the r.v.'s $\left[c_{m-\Phi}^{(k)}-c_{m}^{(1)}\right]$ for distinct values of $m$ are conditionally independent, given the value of the time shift parameter $\Phi$. We also notice that the r.v.'s $\alpha^{(k)}$ for distinct values of $k$ are conditionally independent, given the code $\left\{c_{m}^{(1)}\right\}$ of user one.

Given the signal structure in (2) and the channel conditions, the statistical nature of the MAI in (6) is a function of the node population density within the area of coverage of the WSN.

We now use the data in fig. 2 to illustrate how transmission power savings are possible when the node population density is high enough. Let's look the results for $\mathrm{SIR}=12.4 \mathrm{~dB}$. If MAI satisfy the GA the BER is about $10^{-7}$, but if MAI satisfy the PA then BER is about $10^{-3}$. Let's say we have a target BER of $10^{-3}$. If we are operating in the so called $\mathrm{PA}$ regime, a $\mathrm{SIR}=12.4 \mathrm{~dB}$ is just enough, but if we are operating in the so called GA regime, a lower SIR would be fine.

This means transmitter node 2 could reduce its power transmission and still satisfy the target BER. Hence, if node 2 somehow knows that the GA regime applies, it will proceed to reduce its transmission power. To determine if the GA regime can be applied or not, it would need to know how many nodes are in its own vicinity, i.e., it would need to know the node population density in its neighborhood.

The GA regime is reasonable for SSMA systems with both low per-user data rate and a large number of users. Since WSN transmit at low bit rate and are usually densely populated, transmission power could be controlled according the statistical nature of the MAI produced by the nodes in the close vicinity of the target node.

We now proceed to investigate what would be the critical number of nodes in the vicinity from which the GA regime can be applied. 


\section{Determining the number of nodes to apply the GA regime}

To study how the domain of validity of the GA regime changes we consider 3 scenarios: An ideal propagation channel with perfect and imperfect power control, ${ }^{3}$ as well as a multipath channel with "perfect average" power control. In our method we fix certain signal design parameters (e.g. pulse shape and duration, pulse position and frame time $\left.T_{f}\right)$, and find for which pairs $\left(N_{s}, N_{u}\right)$ the Gaussian assumption can be considered valid using an entropy test to determine Gaussianity.

\subsection{Gaussianity Test.}

In this section we describe a procedure to determine the Gaussianity of a sample $\alpha$ of MAI. More specifically, we collect a sample $\alpha$ in (6) and calculate an entropy function which is then compared with the entropy of a Gaussian r.v.

Let's consider the following hypothesis testing

$$
\begin{aligned}
& \mathcal{H}_{0}: \alpha \sim N\left(0, \sigma^{2}\right) \\
& \mathcal{H}_{1}: \alpha \sim \operatorname{not} N\left(0, \sigma^{2}\right),
\end{aligned}
$$

where $\alpha$ is assumed to have zero-mean and variance $\sigma^{2}$, and $N(\cdot)$ is the normal distribution. $\mathcal{H}_{1}$ say's that the distribution of $\alpha$ is not Gaussian. By exploiting the fundamental fact that a r.v. has maximum differential entropy if and only if its Gaussian distributed [13], the following equivalent hypothesis testing problem can be established:

$$
\begin{aligned}
& \mathcal{H}_{0}: \operatorname{entropy}(\alpha)=\frac{1}{2} \ln \left(2 \pi e \sigma^{2}\right) \\
& \mathcal{H}_{1}: \operatorname{entropy}(\alpha)<\frac{1}{2} \ln \left(2 \pi e \sigma^{2}\right)
\end{aligned},
$$

where $e$ is the base of $\ln (\cdot)$.

An elaboration of hypothesis testing in (8) by [14] gives

$$
\begin{aligned}
& \mathcal{H}_{0}: K_{n m} \rightarrow \sqrt{2 \pi e}=4.1327 \ldots . \\
& \mathcal{H}_{1}: K_{n m}<\sqrt{2 \pi e}
\end{aligned}
$$

where

$$
K_{n m}=\frac{n}{2 m \bar{\sigma}}\left\{\prod_{i=1}^{n}\left(\alpha_{(i+m)}-\alpha_{(i-m)}\right)\right\}^{1 / n}
$$

is a normalized Gaussianity index, and where $\bar{\sigma}^{2}=\frac{1}{n} \sum_{i=1}^{n}\left(\alpha_{i}-\bar{\alpha}\right)^{2} \neq 0$, is the sample variance.

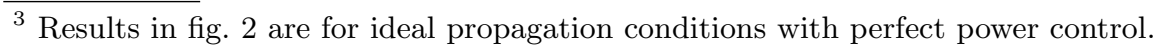


To establish the Gaussianity test a critical value $K^{*}$ is proposed so that when $K_{n m} \geq K^{*}$, with certain probability, we accept that $\alpha$ is a Gaussian r.v. For this purpose we use $K^{*}=3.35$ that has a $95 \%$ confidence level for $n=50$ and $m=5$, and was originally proposed in [14]. With these assumptions we can rewrite the final hypothesis testing problem as

$$
\begin{aligned}
& \mathcal{H}_{0}: K_{n m} \geq K^{*} \\
& \mathcal{H}_{1}: K_{n m}<K^{*} .
\end{aligned}
$$

For a given $p(t)$ and $T_{f}$, we can use this test to define the Gaussian regime as the set of all pair of points $\left(N_{s}, N_{u}\right)$ such that the normality index $K_{n, m} \geq K^{*}$.

\section{Simulation results.}

\section{1 scenarios}

Fig. 3 depicts the power profiles for the three scenarios considered: 1) Ideal propagation with perfect power control, where the power of each component in the MAI is equal to a constant, 2) Ideal propagation with imperfect power control, where the power of each component $k=3,4, \ldots, N_{u}$ in the MAI is a r.v., and 3) A multipath channel with "perfect average" power control, where the power of each node $k=2,3,4, \ldots, N_{u}$ is a r.v. For the random powers we consider multipath channel models [15] [16] with two power profiles: line-ofsight (LOS) and not-line-of-sight (NLOS). We use transmitter-receiver distances $D=3,6,9 \mathrm{~m}$ for LOS and $D=1,2,3 \mathrm{~m}$ for NLOS. We produce 49 profiles for each distance and average over the 3 distances in each case.
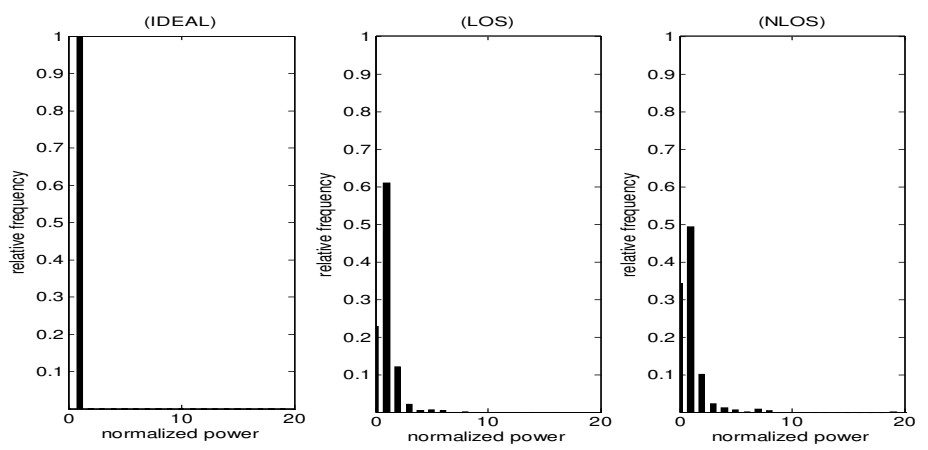

Fig. 3. Power profiles considered in this work. 


\subsection{Calculation of normality index}

Samples of the r.v. $\alpha$ were generated using Matlab. The $\alpha$ in (6) can be seen as a double sum of i.i.d r.v.'s containing terms $R(\cdot)$.

Next we evaluate the normality index $K_{n, m}$ to obtain the region where $\alpha$ is considered a Gaussian r.v., i.e., $K_{50,5} \geq 3.35$. The boundary of the Gaussianity region is determined by those $\left(N_{s}, N_{u}\right)$ where the entropy test is satisfied.

We simulate several cases considering a large amount of permutations of $\left(N_{s}, N_{u}\right)$. Since the entropy estimator is itself a r.v., the random boundary is actually a region determined by many random realizations. To study the boundary with a fixed $T_{f}$ value we generate 100 of such realizations. To study boundary changes for different $T_{f}$ we smooth the plots by averaging over 10 of such realizations.

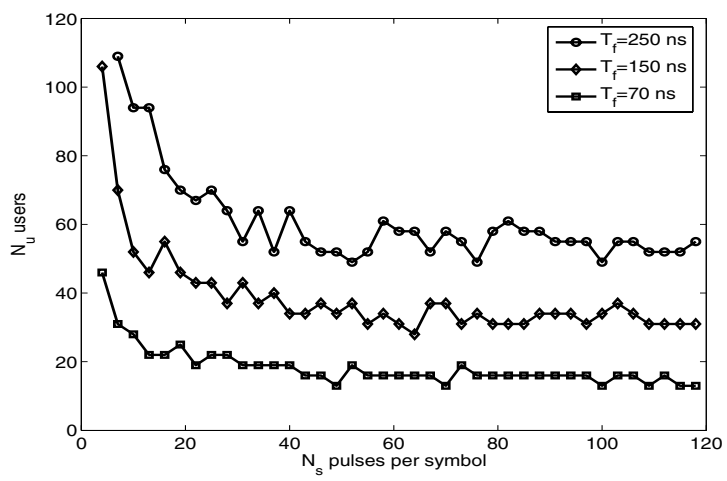

Fig. 4. Boundaries of the Gaussianity region for different $T_{f}$ with perfect power control (Gaussianity region is on top and to the left of the boundary).

\subsection{Ideal propagation with perfect power control}

Fig. 4 shows how the boundary changes for different $T_{f}$ using the Gaussian pulse in (3). Comparing our results with previous works, we found a good match. For example, fig. 2(a) in [11] shows that for $N_{s}=8$ and $T_{f}=150 \mathrm{~ns}$ about $N_{u}=50$ users are needed to be in the Gaussian region, a number that agrees with results in fig. 4. As another example, propositions 6 and 7 in [9] shows that for a fixed $N_{s}$ the larger $T_{f}$ is the larger $N_{u}$ should be to reach Gaussianity, a situation that agrees with results in fig. $4 .^{4}$ Intuitively, a shorter $T_{f}$ results in more pulse

${ }^{4}$ Notice that [9] uses a signal format and parameters in which $N_{s}, N_{h}$ and $T_{f}$ are coupled, i.e., they define $T_{f}=N_{h} T_{c}$ and a processing gain $N=N_{s} N_{h}$, hence $T_{f}=N T_{c} / N_{s}$. By keeping $N$ fixed, a low value of $N_{s}$ implies a large value of $T_{f}$. Notice that in our case we consider PPM with $T_{f} / 2>N_{h} T_{c}+2 T_{d}$, hence $N_{h}$ and $N_{s}$ can be changed without necessarily affecting $T_{f}$ (see fig. 1). 
collisions than a longer $T_{f}$, hence with a shorter $T_{f}$ Gaussianity is achieved with lower values of $N_{s}$ and/or $N_{u}$.
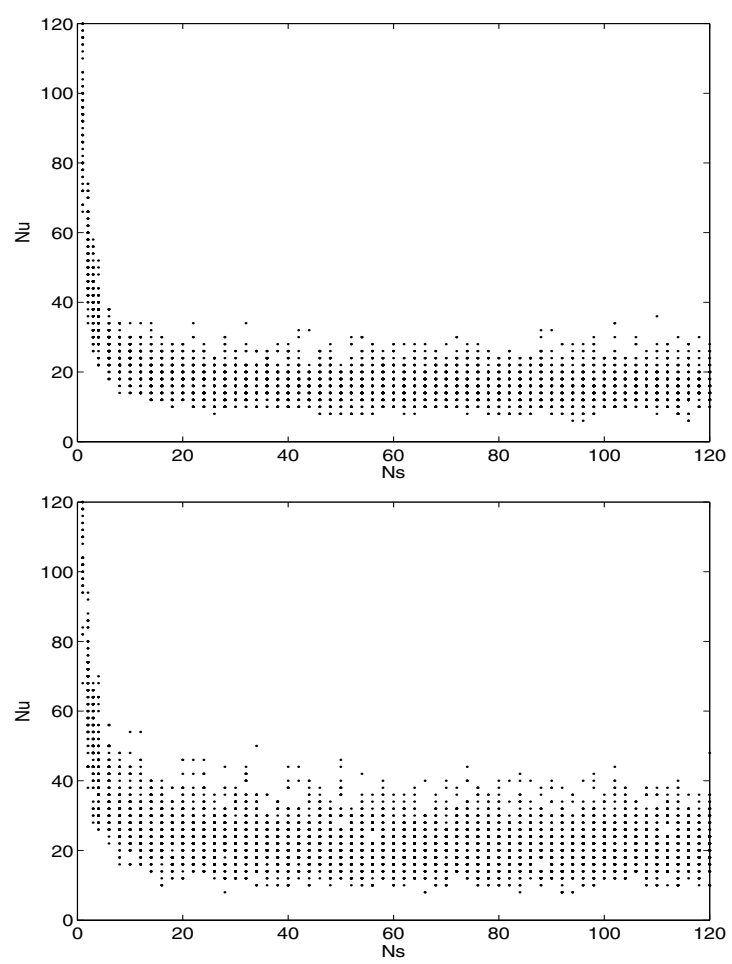

Fig. 5. Boundaries of the Gaussianity region with imperfect power control. Top: LOS power profile. Bottom: NLOS power profile.

\subsection{Ideal propagation with imperfect power control}

Fig. 5 shows the range of pairs $\left(N_{s}, N_{u}\right)$ defining the random boundary using the pulsed sinusoid. We notice that there is an asymmetry on the range of values for $N_{s}$ and $N_{u}$ needed to reach Gaussianity. On one hand, it is observed that for low $N_{s}$ we need a large $N_{u}$ to reach Gaussianity. This can be explained by recalling that $\alpha^{(k)}$ for $k=3,4, \ldots, N_{u}$ are dependant r.v.'s trough user one's code $\left\{c_{m}^{(1)}\right\}$. On the other hand, it is observed that $N_{u}$ reaches a sort of minimum floor as $N_{s}$ is increased. This can be explained recalling that $\lambda_{m}^{(k)}$ for $m=0,2, \ldots, N_{s}-1$ are dependant r.v.'s trough $\Phi$, and that $\tau_{k}$, being uniformly distributed over $\left[-\frac{T_{f}}{2}, \frac{T_{f}}{2}\right]$, is the largest component in $\lambda_{m}^{(k)}$ compared to $\left[c_{m-\Phi}^{(k)}-c_{m}^{(1)}\right] T_{c}+d^{(k)} T_{d}$.

Results for this case also show that the boundary with imperfect power control have a higher spread of $\left(N_{s}, N_{u}\right)$ values than the boundaries with perfect 
power control. This is expected since the weak components of the MAI are masked by the strong ones, hence it takes more r.v. to get to the Gaussianity region. We also notice that boundaries for NLOS have a higher spread than the boundaries for LOS. This is explained observing that the NLOS power profile in fig. 3 have a higher spread than the LOS power profile.
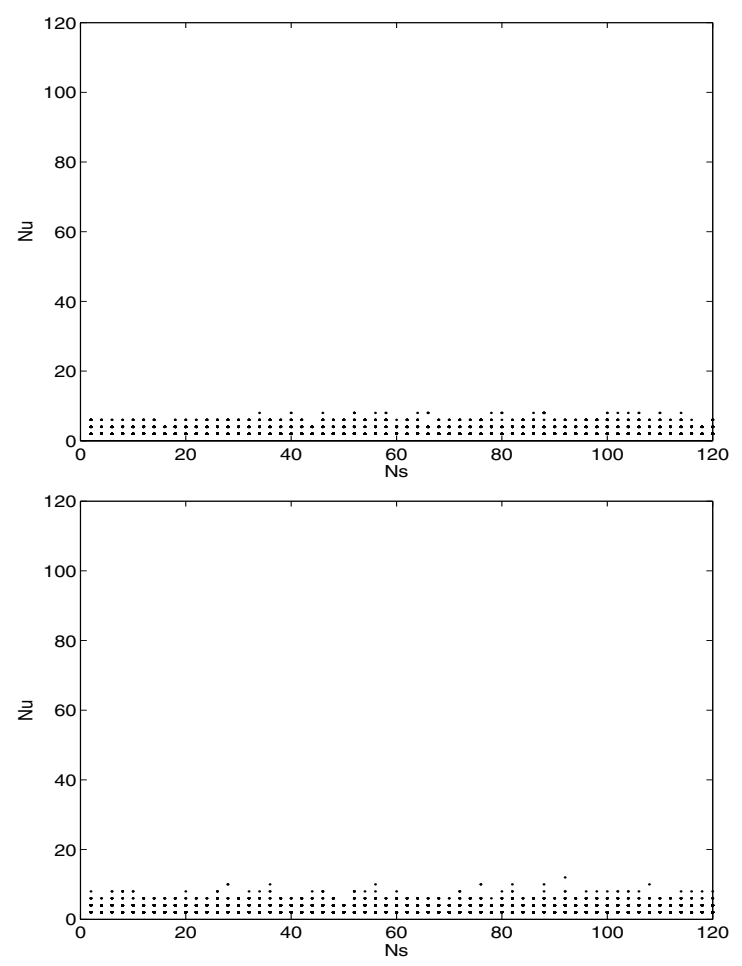

Fig. 6. Boundaries of the Gaussianity region in a multipath channel with average power control. Top for LOS and bottom for NLOS.

\subsection{Multipath channel with perfect average power control}

Fig. 6 shows the random boundary using the pulsed sinusoid in (4). We notice that in this case there is also an asymmetry on the range of values for $N_{s}$ and $N_{u}$ needed to reach Gaussianity, i.e., the range of $N_{u}$ needed to reach Gaussianity is approximately constant for all values of $N_{s}$ considered, and this range contains just a few interferer users, hence, in a dense multipath Gaussianity can be reached with few values of both $N_{s}$ and $N_{u}$. These results are consistent with previous 
studies where a single-user UWB signal in multipath can be modeled as a nonstationary Gaussian random process $[17] .^{5}$

\section{Discussion and conclusions.}

In this paper we propose a method to manage transmission power in nodes belonging to an IR-UWB WSN.

Transmission power is controlled according to the statistical nature of the MAI produced by interfering nodes in the close vicinity of the target node. The statistical nature of the MAI is a function of the node population density within the area of coverage of the WSN.

We established a statistical test to determine when the MAI at the output of a correlator can be considered a r.v. with a Gaussian distribution. Using an entropy point estimator we determine the minimum number of users $N_{u}$ and number of pulses per symbol $N_{s}$ necessary to consider the MAI component as a Gaussian r.v. We show that when the node population density is high enough MAI can be considered as a Gaussian random variable and in that case transmission power savings are possible. Our analysis assumes operation at low to medium SNR values.

One issue (noted by a reviewer) is the mechanism that allows a node to know the spreading code of the other nodes and to know the population density in its vicinity. This power management technique can be used during periods of stability in the network, e.g., once the topology and node population are relatively stable, and after the network initial communication set up has been completed. For the nodes to remain simple, some form of centralized control and monitoring should be used, but further research is needed to find specific protocols that can handle operation in relatively stable conditions as well as during transitions. One potential advantage of our technique relatively to other work in the literature [18] [19] is that we do not require to estimate the power of the interference but just to register the number of the interferers.

Another issue (noted by a reviewer) is whether or not the use of the proposed power-control technique could lead to 'oscillatory' behavior in which the MAI statistics switches back and for from the GA regime to the PA regime as nodes increase and decrease the power levels. Results in fig. 5 for ideal propagation with imperfect power control implicitly consider this situation, ${ }^{6}$ showing that the GA regime is stable for $N u$ high enough (e.g. $N_{u} \geq 60$ nodes in fig. 5). However, this value of $N_{u}$ can change with the statistics of the power profile.

${ }^{5}$ Notice, however, that fig. 7 in [9] shows that a higher $N_{u}$ is needed to reach Gaussianity. But results in [9] are for a multipath channel with a limited number of discrete paths, while in this work we are using a dense multipath channel with a continuous impulse response.

${ }^{6}$ In fig. 5 the received power from the different nodes fluctuates from low to high to low again according to the power profile in fig. 3 . 


\section{References}

1. I. F. Akyildiz, W. Su, Y. Sankarasubramaniam, and E. Cayirci, A survey on sensor networks, in IEEE Communications Magazine, Vol. 40 (2002), pp. 102- 114.

2. Various authors, Special issue on ultra-wideband communications, in IEEE J. Select. Areas Commun., vol. 24 (2006).

3. U.S. Federal Communications Commission, First Report and Order for UWB Technology, U.S. Federal Communications Commission, (2002).

4. R. A. Sholtz, Multiple-access with time-hopping impulse modulation, in Proc. Military Communications Conf. (1993) pp. 447-450.

5. L. De Nardis, P. Baldi, and M.-G. Di Benedetto, UWB Ad-Hoc networks, in IEEE Conference on Ultra Wideband Systems and Technologies, (2002), pp. $219-223$.

6. L. De Nardis, and M.-G. Di Benedetto, Joint communication, ranging, and positioning in low data-rate UWB networks, in 2nd. Workshop on positioning, navigation and communication and 1st ultra-wideband expert talk, (2005), pp. 191-200.

7. F. Ramírez-Mireles, Performance of ultrawideband SSMA using time hopping and $M$-ary PPM, in IEEE J. Select. Areas Commun., vol. 19 (2001), pp. 1186-1196.

8. F. Ramírez-Mireles, Performance of UWB SSMA using orthogonal PPM-TH over dense multipath channels, in Springer's Telecommunications Systems, vol. 36 (2007), pp. 107-115.

9. J. Fiorina and W. Hachem, On the asymptotic distribution of the correlation receiver output for time-hopped UWB signals, in IEEE Trans. on Signal Processing, vol. 54 (2006), pp. 2529 - 2545.

10. N. C. Beaulieu and S. Niranjayan, New UWB Receiver Designs Based on a Gaussian-Laplacian Noise-Plus-MAI Model, in IEEE International Conference on Communications, (2007), pp. 4128 - 4133.

11. Y. Dhibi and T. Kaiser, On the impulsiveness of multiuser interferences in THPPM-UWB systems, in IEEE Trans. on Signal Processing, vol. 54 (2006), pp. 2853 $-2857$.

12. A. Papoulis, Probability, random variables, and stochastic processes, New York:. McGraw Hill (1965).

13. Robert B. Ash, Information theory, Dover Publications, New York, (1990).

14. O. Vasicek, A test for normality based on sample entropy, Journal of the Royal Statistical Society Series B, vol. 38 (1976), pp. 54-59, 1976.

15. W. Turin, R. Jana, S. Ghassemzadeh, C. Rice, and V. Tarokh, Autoregressive modeling of an indoor UWB channel, in Proc. UWBST Conf.,(2002), pp. 71-74.

16. S. Ghassemzadeh, R. Jana, C. Rice, W. Turin, and V. Tarokh, A statistical path loss model for in-home UWB channel, in Proc. UWBST Conf., (2002), pp. 59-64.

17. Q. T. Zhang and S. H. Song, "Parsimonious Correlated Nonstationary Models for Real Baseband UWB Data", in IEEE Trans. on Vehic. Technol., vol. 54, pp. 447-455, Mar. 2005.

18. F. Cuomo, C. Martello, S. Baiocchi, F. Capriotti, "Radio Resource Sharing for Ad Hoc Networking with UWB", in IEEE JSAC, vol. 20 (2002), pp. 1722-1732.

19. H. Jiang, W. Zhuang, and X. Shen, "Distributed medium access control for next generation CDMA wireless networks", in IEEE Wireless Communications, vol. 14 (2007), pp. 25-31. 